\title{
Application of the Homotopy Perturbation Method (HPM) and the Homotopy Analysis Method (HAM) to the Problem of the Thermal Explosion in a Radiation Gas with Polydisperse Fuel Spray
}

\section{Ophir Nave ${ }^{1 *}$, Yaron Lehavi ${ }^{2}$, Vladimir Gol'dshtein ${ }^{1}$ and Suraj Ajadi ${ }^{3}$}

${ }^{1}$ Department of Mathematics, Ben-Gurion University of the Negev, PO Box 653, Beer-Sheva, 84105, Israel

2Jerusalem College of Technology (JCT), Department of Physics David Yellin, Jerusalem, Israel

${ }^{3}$ Faculty of Science, Department of Mathematics, O.A.U, Ile-Ife, Nigeria

\begin{abstract}
The aim of this work is to apply the homotopy perturbation method and homotopy analysis method to the problem of thermal explosion in a flammable gas mixture with the addition of volatile fuel droplets. The system of equations that describes the effects of heating, evaporation, and combustion of fuel in a polydisperse spray is simplified. Both convective and radiative heating of droplets is taken into account in the model. The model for the radiative heating of droplets takes into account the semitransparency of the droplets. The results of the analysis have been applied to the modeling of the thermal explosion in diesel engines. We applied the Homotopy Perturbation Method and the Homotopy Analysis Method to the new model and we found the region of the convergence of the considered solutions of the relevant physical parameters. The results demonstrate that these methods are very effective for solving nonlinear problems in science and engineering.
\end{abstract}

Keywords: Homotopy perturbation method (HPM); Homotopy analysis method (HAM); Nonlinear differential equations; Thermal explosion; Polydisperse fuel spray

\section{Nomenclature}

- $A$ pre-exponential rate factor $\left(S^{-1}\right)$

- $B$ universal gas constant $\left(\mathrm{Jkmol}^{-1} \mathrm{~K}^{-1}\right)$

- $C$ molar concentration $\left(\mathrm{kmolm}^{-3}\right)$

- $C$ specific heat capacity $\left.\left(\mathrm{Jkg}^{-1} \mathrm{~K}^{-1}\right)\right)$

- $E$ activation energy $\left(\mathrm{Jkmol}^{-1}\right)$

- $F-M$ refer to the model (21)-(24) with $b=0$

- $k$ number of droplets

- $L$ liquid evaporation energy (i.e., latent heat of evaporation, Enthalpy of evaporation) $\left(\mathrm{Jkg}^{-1}\right)$

- $m$ droplet mass

- $n$ number of droplets per unit volume $\left(\mathrm{m}^{-3}\right)$

- $Q$ combustion energy ( $\left.\mathrm{Jkg}^{-1}\right)$

- $q$ heat flux $\left(\mathrm{Wm}^{-2}\right)$

- $R$ radius of droplet $(m)$

- $r$ dimensionless radius

- $T$ temperature ( $K$ )

- $t$ time $(S$ )

- $t_{\text {react }}$ characteristic reaction time ( $S$ ) defined in Equation (25)

Greek symbols

- $\alpha$ dimensionless volumetric phase content

- $\beta$ dimensionless reduced initial temperature (with respect to the so-called activation temperature $E / B$ )

- $\gamma$ dimensionless parameter that represents the reciprocal of the final dimensionless adiabatic temperature of the thermally insulated system after the explosion has been completed

- $\varepsilon_{1 i} i=1, \ldots, k$ dimensionless parameters defined in Equation (25) and describes the competition between the combustion and the evaporation processes
- $\varepsilon_{2 i} i=1, \ldots, k$ dimensionless parameters defined in Equation (25). This parameter relates the heat released during combustion and energy that is needed to evaporate all the fuel droplets

- $\varepsilon_{3 i} i=1, \ldots, k$ dimensionless parameters defined in Equation (25). This parameter describes the ratio of $t_{\text {react }}$ and the characteristic droplet heating time

- $\varepsilon_{4 i} i=1, \ldots, k$ dimensionless parameters defined in Equation (25). This parameter is proportional to the ratio of radiative and convective fluxes

$\eta$ dimensionless fuel concentration

- $\theta$ dimensionless temperature

- $\lambda$ thermal conductivity $\left(W^{-1} K^{-1}\right)$

- $\mu$ molar mass $\left(\mathrm{kgkmol}^{-1}\right)$

- $v$ dimensionless parameter defined in Equation (25)

- $\rho$ density $\left(\mathrm{kgm}^{-3}\right)$

- $\sigma$ Stefan-Boltzmann constant $\left(\begin{array}{ll}W & m^{-2} K^{-4}\end{array}\right)$

- $\tau$ dimensionless time

- $\psi$ represents the internal characteristics of the fuel (the ratio of the specific combustion energy and the latent heat of evaporation) defined in Equation (25) and for diesel fuel $\psi>>1$

\section{Subscripts}

- con convection

*Corresponding author: Ophir Nave, Department of Mathematics, Ben-Gurion University of the Negev, PO Box 653, Beer-Sheva, 84105, Israel, Fax: 972-8-6477648, E-mail: naveof@bgu.ac.il

Received April 20, 2012; Accepted June 20, 2012; Published June 23, 2012

Citation: Nave O, Lehavi Y, Gol'dshtein V, Ajadi S (2012) Application of the Homotopy Perturbation Method (HPM) and the Homotopy Analysis Method (HAM) to the Problem of the Thermal Explosion in a Radiation Gas with Polydisperse Fue Spray. J Appl Computat Math 1:115. doi:10.4172/2168-9679.1000115

Copyright: ( 2012 Nave O, et al. This is an open-access article distributed under the terms of the Creative Commons Attribution License, which permits unrestricted use, distribution, and reproduction in any medium, provided the original author and source are credited. 
Citation: Nave O, Lehavi Y, Gol'dshtein V, Ajadi S (2012) Application of the Homotopy Perturbation Method (HPM) and the Homotopy Analysis Method (HAM) to the Problem of the Thermal Explosion in a Radiation Gas with Polydisperse Fuel Spray. J Appl Computat Math 1:115. doi:10.4172/2168-9679.1000115

Page 2 of 9

- $d$ liquid fuel droplets

- $f$ combustible gas component of the mixture

- $g$ gas mixture

- $l$ liquid phase

- $p$ under constant pressure

- rad adiation

- 0 initial state

\section{Introduction}

In this paper we investigated the problem of thermal explosion in a fuel mixture and gas. This problem, in most cases, has been studied based on the application of computational fluid dynamics (CFD) packages [1]. This method could take into account the complicated geometry of the enclosure and the chemistry of the processes. Hence, this makes it particularly attractive for engineering applications including the modeling of combustion processes in diesel engines. Other approaches to this problem are based on a asymptotic analysis of equations describing the limiting cases of the processes. One of these approaches is based on the application of the zero-order approximation of the geometric version of the asymptotic method of integral manifolds (MIM), developed for combustion applications in [2,3]. For example, in [4] the method of integral manifold was applied to a specific problem of modeling of ignition process in a diesel engines by using the P-1 model. The chemical term was presented in the Arrhenius form with the pre-exponential factor calculated from the enthalpy equation, using the well known Shell autoignition model. The results predicted by the analytical solution were compared with those that predicted by the computational fluid dynamics package VECTIS. The effects of the thermal radiation were shown to be very significant especially at high temperatures.

Other asymptotic methods were proposed by [5] in 1992, known as the homotopy analysis method (HAM) and by [6] in 1998, known as the homotopy perturbation method (HPM), which is a special case of HAM. The HPM and the HAM methods are mathematical tools that are based on homotopy, a fundamental concept in topology and differential geometry. They are analytical approaches to formulate the series solution of linear and nonlinear partial differential equations. We refer the reader to [7] for an enlightening comparison between HAM and HPM.

HPM couples the homotopy technology and perturbation method including the modified Lindstedt-Poincare method [8]. The authors of [9] modified the Multiple Scales method by incorporating the time transformation of Lindstedt Poincare method. In [10] the authors contrasted two different approaches of Lindstedt-Poincare methods using the duffing equation. The main deficiencies in applying perturbation methods is that a small parameter is needed in the equations.

The HPM was further developed and improved and applied to nonlinear oscillators with discontinuities [11], nonlinear wave equations [12], boundary value problem [13], limit cycle and bifurcation of nonlinear problems [14] and many other subjects. In recent years, the application of the homotopy perturbation method (HPM) in nonlinear problems has been developed by scientists and engineers, because this method deforms the difficult problem under study into a simple problem which is easy to solve [15-17]. Most perturbation methods assume a small parameter exists, but most nonlinear problems have no small parameter at all. Unlike analytical perturbation methods, the
HPM and HAM do not depend on a small parameter which is difficult to find.

These two methods also provide a simple way to ensure the convergence of the series solution. Moreover, these methods provide a large degree of freedom to choose an appropriate base functions to approximate the linear and nonlinear problems [18]. Another important advantage of this method is that one can construct a continuous mapping of an initial guess approximation to the exact solution of the given problem through an auxiliary linear operator. To ensure the convergence of the series solution an auxiliary parameter is used. In [19] Liao has substantiated that the HAM differs from the other analytical methods in that it ensures the convergence of the series solution by choosing a proper value for the convergence-control parameter.

In this paper we have rewritten the model that was proposed by [20] for polydisperse fuel spray and applied the HPM and HAM to the problem of thermal explosion in a fuel mixture and gas. Based on these two methods, we present an analytical solutions for various values of the relevant physical parameter and we discuss the convergence of these solutions. We also compare our results to numerical solutions.

\section{An Introduction to the Homotopy Perturbation Method (HPM)}

To explain this method, let us consider the following equation:

$$
A(u)-f(r)=0, \quad r \in \Omega,
$$

with the boundary conditions of:

$$
B\left(u, \frac{\partial u}{\partial n}\right)=0,
$$

where $A, B, f(r)$ are a general differential operator, a boundary

operator, a known analytical function respectively. $\Omega$ is the domain. Generally, the operator $A$ can be decomposed into a linear part $L$ and a nonlinear part $N(u)$. Hence, Equation (1) can be written as:

$$
L(u)+N(u)-f(r)=0 .
$$

By the homotopy technique, we construct a homotopy $\alpha(r, p): \Omega \times[0,1] \rightarrow R$ which satisfies:

$\operatorname{blueH}(\alpha(r, p), p)=(1-p)\left(L(\alpha(r, p))-u_{0}\right)+p(A(\alpha(r, p))-f(r))=0, \quad p \in[0,1], r \in \Omega$,

where $p \in[0,1]$ is an embedding parameter and $\alpha(r, p)$ is a function of $r$ and $p$, and $u_{0}(r)$ denote the initial approximation of $\alpha(r)$.

When $p=0$ we have

$$
\operatorname{blueH}(\alpha(r, p), p)_{\mid p=0}=\left(L(\alpha)-u_{0}\right),
$$

and when $p=1$ we have

$$
\operatorname{blueH}(\alpha(r, p), p)=A(\alpha(t, p))-f(r) .
$$

As we mention before, $L$ denote an auxiliary linear operator. In addition $L$ have the property:

$$
\operatorname{blueL}(g)=0 \text { for } \quad g=0 .
$$

Using (7), it is clear that for $p=0$

$$
\text { bluea }(r, 0)=u_{0}(r)
$$


Citation: Nave O, Lehavi Y, Gol'dshtein V, Ajadi S (2012) Application of the Homotopy Perturbation Method (HPM) and the Homotopy Analysis Method (HAM) to the Problem of the Thermal Explosion in a Radiation Gas with Polydisperse Fuel Spray. J Appl Computat Math 1:115. doi:10.4172/2168-9679.1000115

Page 3 of 9

is the solution of the equation:

$$
\operatorname{blueH}(\alpha(r, p), p)_{p=0}=0 .
$$

And for $p=1$

$$
\operatorname{blue} \alpha(r, 1)=u(r)
$$

is the solution of the equation:

$$
\operatorname{blueH}(\alpha(r, p), p)_{p=1}=0 .
$$

When the embedding parameter $p$ increase from 0 to 1 , the solution $\alpha(r, p)$ of the equation:

$$
H(\alpha(r, p), p)=0
$$

depends upon the embedding parameter $p$ and the varies from the initial approximation $u_{0}(r)$ to the solution $u(r)$ of equation (1). In topology, such a kind of continuous variation is called deformation.

According to the HPM, we can first use the embedding parameter $p$ as a "small parameter", and assume that the solutions of Equation (4) can be written as a power series in $p$ :

$$
\alpha=\alpha_{0}+\alpha_{1} p+\alpha_{2} p^{2}+\ldots=\sum_{m=0}^{\infty} \alpha_{m} p^{m} .
$$

Setting $p=1$ yields in the approximate solution of (1) to

$$
u=\lim _{p \rightarrow 1} \alpha=\alpha_{0}+\alpha_{1}+\alpha_{2}+\ldots=\sum_{m=0}^{\infty} \alpha_{m} .
$$

The combination of the perturbation method and the homotopy method is called the HPM, which eliminates the drawbacks of the traditional perturbation methods while keeping all their advantages. The rate of convergent of series (13) depends on the nonlinear operator $A(u)$.

\section{Polydisperse Model-Problem Statement}

The physical assumptions are as follows: The combustible gas mixture contains evaporating ideal spherical droplets of fuel. The liquid droplets form a polydisperse spray. The medium is assumed to be spatially homogeneous. The variations in pressure in the enclosure, and their influence on the combustion processes are ignored. The heat flux from the burning gas to the droplets is assumed to consist of two components: convection and radiation, and the form of these two components is as follows:

$$
\begin{gathered}
q_{\text {con }}=\frac{\lambda_{g}}{R_{d_{i}}}\left(T_{g}-T_{d_{i}}\right), \quad \lambda_{g}=\sqrt{\frac{T_{g}}{T_{g 0}}}, \\
q_{\text {rad }}=\sigma R_{d_{i}}^{b}\left(10^{3} k_{01}-k_{11} T_{g}\right)\left(T_{g}^{4}-T_{d_{i}}^{4}\right),
\end{gathered}
$$

where for $\mu m$ units the value of $k_{01}$ and $k_{11}$ are: $k_{01}=7 \cdot 10^{-5}$ and $k_{11}=2 \cdot 10^{-2} K^{-1}$. The energy that is needed for heating fuel vapor from the droplet temperature to gas temperature is ignored. The thermal conductivity of the liquid phase is much greater than that of the gas phase. The volume fraction of the liquid phase is much less than that of the gas phase. The heat transfer coefficient in the liquidgas mixture is assumed to be controlled by the thermal properties of the gas phase. External heat losses are ignored. Fuel droplets are semitransparent. Combustion takes place in the gas phase only. Combustion is modeled as a one-step first-order exothermic reaction with gaseous fuel as a deficient reactant. The droplets are assumed to be stationary.
Under these assumptions, we rewrite the model as in [21], which is in the form of monodisperse fuel spray, to a polydisperse fuel spray as follows:

$$
\begin{gathered}
c_{p g} \rho_{g} \alpha_{g} \frac{d T_{g}}{d t}=C_{f} Q_{f} \mu_{f} \alpha_{g} A e^{-\left(\frac{E}{B T_{g}}\right)}-4 \pi \sum_{i=1}^{k} R_{d_{i}}^{2} n_{d_{i}}\left(q_{c o n}+q_{r a d}\right), \\
\alpha_{g} \frac{d C_{f}}{d t}=-C_{f} \alpha_{g} A e^{-\left(\frac{E}{B T_{g}}\right)}+4 \pi \sum_{i=1}^{k} \frac{R_{d_{i}}^{2} n_{d_{i}}}{L \mu_{f}}\left(q_{c o n}+q_{r a d}\right), \\
C_{d_{i}} m_{d_{i}} \frac{d T_{d_{i}}}{d t}=4 \pi R_{d_{i}}{ }^{2}\left(q_{c o n}+q_{r a d}\right), \quad i=1, \ldots, k, \\
\frac{d m_{d_{i}}}{d t}=-\frac{4 \pi R_{d_{i}}^{2}}{L}\left(q_{c o n}+q_{r a d}\right), \quad i=1, \ldots, k .
\end{gathered}
$$

In non-dimensional parameters and by applying the FrankKamenetskii approximation [22], the model has the form of:

$$
\begin{aligned}
& \frac{d \theta_{g}}{d \tau}=\gamma^{-1} \eta e^{\theta_{g}}-\gamma^{-1} \sum_{i=1}^{k} \varepsilon_{1 i} r_{d_{i}}\left(\theta_{g}-\theta_{d_{i}}\right)\left(1+\varepsilon_{4 i}(v-1) r_{d_{i}}^{b+1}\right), \\
& \frac{d \eta}{d \tau}=-\eta e^{\theta_{g}}+\psi \sum_{i=1}^{k} \varepsilon_{1 i} r_{d_{i}}\left(\theta_{g}-\theta_{d_{i}}\right)\left(1+\varepsilon_{4 i}(v-1) r_{d_{i}}^{b+1}\right), \\
& \frac{d \theta_{d_{i}}}{d \tau}=\varepsilon_{3 i} r_{d_{i}}\left(\theta_{g}-\theta_{d_{i}}\right)\left(1+\varepsilon_{4 i}(v-1) r_{d_{i}}^{b+1}\right), \\
& \frac{d r_{d_{i}}^{3}}{d \tau}=-\varepsilon_{1 i} \varepsilon_{2 i} r_{d_{i}}\left(\theta_{g}-\theta_{d_{i}}\right)\left(1+\varepsilon_{4 i}(v-1) r_{d_{i}}^{b+1}\right),
\end{aligned}
$$

where the following dimensionless parameters have been introduced:

$$
\begin{gathered}
\beta=\frac{B T_{g 0}}{E}, \quad \tau=\frac{t}{t_{\text {react }}}, \nu=\frac{k_{01} 10^{3}}{k_{11} T_{g 0}}, \quad t_{\text {react }}=\frac{e^{1 / \beta}}{A}, \\
r_{i}=\frac{R_{d_{i}}}{R_{d_{i 0}}}, \quad \gamma=\beta \frac{c_{p g} T_{g 0} \rho_{g}}{Q_{f} \mu_{f} C_{f 0}}, \quad \psi=\frac{Q_{f}}{L}, \quad \theta_{g}=\frac{1}{\beta} \frac{T_{g}-T_{g 0}}{T_{g 0}}, \\
\eta=\frac{C_{f}}{C_{f 0}}, \quad \varepsilon_{1 i}=\frac{4 \pi \lambda_{g 0} R_{d i 0} \beta T_{g 0} n_{d i}}{A Q_{f} C_{f 0} \alpha_{g} \mu_{f}} e^{\left(\frac{1}{\beta}\right)}, \quad \varepsilon_{2 i}=\frac{3 C_{f 0 f} \mu_{f} \alpha_{g}}{4 \pi R_{d_{i}}^{3} n_{d_{i}} \rho_{d_{i}} L}, \\
\varepsilon_{3 i}=\frac{3 \lambda_{g 0} e^{1 / \beta}}{A C_{d_{i}} \rho_{d_{i}} R_{d_{i o}}^{2}}, \quad \varepsilon_{4 i}=\frac{4 \sigma k_{11} T_{g 0}^{4} R_{d_{i 0}}^{b+1}}{\lambda_{g 0}}, \quad \theta_{d_{i}}=\frac{T_{d_{i}}-T_{g}}{\beta T_{g 0}} .
\end{gathered}
$$

The non-dimensional initial conditions are:

$$
\text { at } \quad \tau=0: \quad \theta_{g}=0, \quad \theta_{d_{i}}=\theta_{d_{i 0}}, \quad \eta=\eta_{0}, \quad r_{i}=1 \text {. }
$$

For simplicity, in our numerical simulations and when applying the HPM and the HAM we assume $b=0$

\section{Application of HPM to the Problem of the Thermal Explosion in Polydisperse Fuel Spray}

By applying the HPM method to the system of Equations (21)-(24) we obtain the following HPM-system: 
Citation: Nave O, Lehavi Y, Gol'dshtein V, Ajadi S (2012) Application of the Homotopy Perturbation Method (HPM) and the Homotopy Analysis Method (HAM) to the Problem of the Thermal Explosion in a Radiation Gas with Polydisperse Fuel Spray. J Appl Computat Math 1:115. doi:10.4172/2168-9679.1000115

Page 4 of 9

$$
\begin{gathered}
(1-p)\left(\frac{d \alpha_{1}}{d \tau}-\frac{d \theta_{g}(0)}{d \tau}\right)+p\left(\frac{d \alpha_{1}}{d \tau}-\gamma^{-1} \alpha_{2} e^{\alpha_{1}}\right) \\
+p\left(\gamma^{-1} \sum_{i=1}^{k} \varepsilon_{1 i} \alpha_{4}^{i}\left(\alpha_{1}-\alpha_{3}^{i}\right)\left(1+\varepsilon_{4 i}(v-1) \alpha_{4}^{i}\right)\right)=0 \\
(1-p)\left(\frac{d \alpha_{2}}{d \tau}-\frac{d \eta(0)}{d \tau}\right)+p\left(\frac{d \alpha_{2}}{d \tau}+\alpha_{2} e^{\alpha_{1}}\right) \\
+p\left(-\psi \sum_{i=1}^{k} \varepsilon_{1 i} \alpha_{4}^{i}\left(\alpha_{1}-\alpha_{3}^{i}\right)\left(1+\varepsilon_{4 i}(v-1) \alpha_{4}^{i}\right)\right)=0 \\
(1-p)\left(\frac{d \alpha_{3}^{i}}{d \tau}-\frac{d \theta_{d_{i}}(0)}{d \tau}\right) \\
+p\left(\frac{d \alpha_{3}^{i}}{d \tau}-\varepsilon_{3 i} \alpha_{4}^{i}\left(\alpha_{1}-\alpha_{3}^{i}\right)\left(1+\varepsilon_{4 i}(v-1) \alpha_{4}^{i}\right)\right)=0 \\
1 \leq i \leq k
\end{gathered}
$$$$
(1-p)\left(\frac{d\left(\alpha_{4}^{i}\right)^{3}}{d \tau}-\frac{d r_{d_{i}}(0)}{d \tau}\right)
$$$$
\left.+p\left(\frac{d\left(\alpha_{4}^{i}\right)^{3}}{d \tau}+\varepsilon_{\text {üfi } i} \alpha^{i}\right)\left(\alpha-\alpha^{i}\right)\left(1+\varepsilon_{i}(v-1) \alpha^{i}\right)\right)=0,
$$$$
1 \leq i \leq k
$$

with the initial conditions:

$$
\alpha_{1,0}=\theta_{g}(0)=0, \quad \alpha_{2,0}=\eta(0), \quad \alpha_{3,0}^{i}=\theta_{d_{i}}(0), \quad \alpha_{4,0}^{i}=r_{d_{i 0}} .
$$

According to HPM method the terms $\alpha_{1}, \alpha_{2} \quad \alpha_{3}^{i}$ and $\alpha_{4}^{i}$ for $i=1, \ldots, k$ has the form of:

$$
\begin{gathered}
\alpha_{1}=\sum_{m=0}^{\infty} \alpha_{1, m}(\tau) p^{m}, \quad \alpha_{2}=\sum_{m=0}^{\infty} \alpha_{2, m}(\tau) p^{m} \\
\alpha_{3}^{i}=\sum_{m=0}^{\infty} \alpha_{3, m}^{i}(\tau) p^{m}, \quad \alpha_{4}^{i}=\sum_{m=0}^{\infty} \alpha_{4, m}^{i}(\tau) p^{m} \quad i=1, \ldots, k .
\end{gathered}
$$

Suppose that the solution of (27)-(30) takes the form of

$$
\begin{aligned}
& \theta_{g}=\lim _{p \rightarrow 1}\left(\sum_{m=0}^{\infty} \alpha_{1, m} p^{m}\right)=\alpha_{1,0}+\alpha_{1,1}+\alpha_{1,2}+\alpha_{1,3}+\ldots, \\
& \eta=\lim _{p \rightarrow 1}\left(\sum_{m=0}^{\infty} \alpha_{2, m} p^{m}\right)=\alpha_{2,0}+\alpha_{2,1}+\alpha_{2,2}+\alpha_{2,3}+\ldots, \\
& \theta_{d_{i}}=\lim _{p \rightarrow 1}\left(\sum_{m=0}^{\infty} \alpha_{3, m}^{i} p^{m}\right)=\alpha_{3,0}^{i}+\alpha_{3,1}^{i}+\alpha_{3,2}^{i}+\alpha_{3,3}^{i}+\ldots(i=1, \ldots, k), \\
& r_{i}=\lim _{p \rightarrow 1}\left(\sum_{m=0}^{\infty} \alpha_{4, m}^{i} p^{m}\right)=\alpha_{4,0}^{i}+\alpha_{4,1}^{i}+\alpha_{4,2}^{i}+\alpha_{4,3}^{i}+\ldots(i=1, \ldots, k) .
\end{aligned}
$$

Substituting Equations (32) with the initial conditions (31) into Equations (27)-(30) for three different size of droplets i.e., $k=3$, using the Taylor expansion for the exponent $[23,24]$ and finally collecting the terms in power of $p$ up to order 3 we obtain:

$$
\begin{gathered}
\text { equations for gas temperature } \theta_{g} \\
\frac{d \alpha_{1,1}}{d \tau}-\gamma^{-1} \alpha_{2,0}=0, \\
\frac{d \alpha_{1,2}}{d \tau}-\gamma^{-1} \alpha_{2,0} \alpha_{1,1}-\gamma^{-1} \alpha_{2,1}+\gamma^{-1} \sum_{i=1}^{3} \varepsilon_{1 i} \alpha_{1,1}+\varepsilon_{1 i} \varepsilon_{4 i}(v-1) \alpha_{1,1} \\
-\varepsilon_{1 i} \alpha_{3,1}^{i}-\varepsilon_{1 i} \varepsilon_{4 i}(v-1) \alpha_{3,1}^{i}=0, \\
\frac{d \alpha_{1,3}}{d \tau}-\gamma^{-1} \alpha_{2,0} \alpha_{1,2}-\gamma^{-1} \alpha_{2,1} \alpha_{1,1}-\gamma^{-1} \alpha_{2,2}+\gamma^{-1} \sum_{i=1}^{3} \varepsilon_{1 i} \alpha_{1,1} \alpha_{4,1}^{i} \\
+\varepsilon_{1 i} \alpha_{1,2}+\varepsilon_{1 i} \varepsilon_{4 i}(v-1)\left\{\left(\alpha_{1,1} \alpha_{4,1}^{i}+\alpha_{1,2}-2 \alpha_{3,1}^{i} \alpha_{4,1}^{i}-\alpha_{3,2}^{i}\right\}\right. \\
-\varepsilon_{1 i} \alpha_{3,1}^{i} \alpha_{4,1}^{i}-\varepsilon_{1 i} \alpha_{3,2}=0,
\end{gathered}
$$

equations for concentration $\eta$

$$
\frac{d \alpha_{2,1}}{d \tau}+\alpha_{2,0}=0
$$$$
\frac{d \alpha_{2,2}}{d \tau}+\alpha_{2,0} \alpha_{1,1}+\alpha_{2,1}-\psi \sum_{i=1}^{3} \varepsilon_{1 i} \alpha_{1,1}+\varepsilon_{1 i} \varepsilon_{4 i}(v-1) \alpha_{1,1}
$$$$
-\varepsilon_{1 i} \alpha_{3,1}^{i}-\varepsilon_{1 i} \varepsilon_{4 i}(v-1) \alpha_{3,1}^{i}=0 \text {, }
$$

$$
\begin{aligned}
& \frac{d \alpha_{2,3}}{d \tau}+\alpha_{2,0} \alpha_{1,2}+\alpha_{2,1} \alpha_{1,1}+\alpha_{2,2}-\psi \sum_{i=1}^{3} \varepsilon_{1 i} \alpha_{1,1} \alpha_{4,1}^{i} \\
& +\varepsilon_{1 i} \alpha_{1,2}+\varepsilon_{1 i} \varepsilon_{4 i}(v-1)\left\{\left(\alpha_{1,1} \alpha_{4,1}^{i}+\alpha_{1,2}-2 \alpha_{3,1}^{i} \alpha_{4,1}^{i}-\alpha_{3,2}^{i}\right\}\right. \\
& -\varepsilon_{1 i} \alpha_{3,1}^{i} \alpha_{4,1}^{i}-\varepsilon_{1 i} \alpha_{3,2}=0,
\end{aligned}
$$

equations for the droplet temperature $\theta_{d_{i}}$

$$
\frac{d \alpha_{3,1}^{i}}{d \tau}=0, \quad(1 \leq i \leq 3)
$$

$$
\begin{aligned}
& \frac{d \alpha_{3,2}^{i}}{d \tau}+2 \frac{d \alpha_{3,1}^{i}}{d \tau}-\varepsilon_{3 i}\left\{\alpha_{1,1}+\varepsilon_{4 i}(v-1)\left(\alpha_{1,1}-\alpha_{3,1}^{i}\right) \alpha_{3,1}^{i}\right\}=0, \\
& (1 \leq i \leq 3)
\end{aligned}
$$

$$
\begin{aligned}
& \frac{d \alpha_{3,3}^{i}}{d \tau}+2 \frac{d \alpha_{3,2}^{i}}{d \tau}-\varepsilon_{3 i}\left(\alpha_{1,1} \alpha_{4,1}^{i}+\alpha_{1,2}+\alpha_{3,1}^{i} \alpha_{4,1}^{i}-\varepsilon_{3 i} \alpha_{3,2}^{i}\right) \\
& +\varepsilon_{3 i} \varepsilon_{4 i}(v-1)\left\{\alpha_{1,1} \alpha_{4,1}^{i}+\alpha_{1,2}-2 \alpha_{3,1}^{i} \alpha_{4,1}^{i}-\alpha_{3,2}^{i}\right\}=0,
\end{aligned}
$$$$
(1 \leq i \leq 3)
$$ 
Citation: Nave O, Lehavi Y, Gol'dshtein V, Ajadi S (2012) Application of the Homotopy Perturbation Method (HPM) and the Homotopy Analysis Method (HAM) to the Problem of the Thermal Explosion in a Radiation Gas with Polydisperse Fuel Spray. J Appl Computat Math 1:115. doi:10.4172/2168-9679.1000115

Page 5 of 9

$$
\begin{aligned}
& \text { equations for the radius } r_{d_{i}} \\
& \frac{d \alpha_{4,1}^{i}}{d \tau}=0, \quad(1 \leq i \leq 3) \\
& \frac{d \alpha_{4,2}^{i}}{d \tau}+2 \frac{d \alpha_{4,1}^{i}}{d \tau}-\varepsilon_{1 i} \varepsilon_{2 i}\left\{\alpha_{1,1}+\varepsilon_{4 i}(v-1)\left(\alpha_{1,1}-\alpha_{3,1}^{i}\right)-\alpha_{3,1}^{i}\right\}=0, \\
& (1 \leq i \leq 3) \\
& \frac{d \alpha_{4,3}^{i}}{d \tau}+2 \frac{d \alpha_{4,2}^{i}}{d \tau}-\varepsilon_{1 i} \varepsilon_{2 i}\left(\alpha_{1,1} \alpha_{4,1}^{i}+\alpha_{1,2}+\alpha_{3,1}^{i} \alpha_{4,1}^{i}-\alpha_{3,2}^{i}\right) \\
& +\varepsilon_{1 i} \varepsilon_{2 i} \varepsilon_{4 i}(v-1)\left\{\alpha_{1,1} \alpha_{4,1}^{i}+\alpha_{1,2}-2 \alpha_{3,1}^{i} \alpha_{4,1}^{i}-\alpha_{3,2}^{i}\right\}=0, \\
& (1 \leq i \leq 3)
\end{aligned}
$$

We derived a system of (24) ordinary differential equation with 24 with unknown functions: $\alpha_{1,1}, \alpha_{1,2}, \alpha_{1,3}, \alpha_{2,1}, \alpha_{2,2}, \alpha_{2,3}, \alpha_{3,1}^{1}, \alpha_{3,2}^{1}, \alpha_{3,3}^{1}$ $, \alpha_{3,1}^{2}, \alpha_{3,2}^{2}, \alpha_{3,3}^{2}, \alpha_{3,1}^{3}, \alpha_{3,2}^{3}, \alpha_{3,3}^{3}, \alpha_{4,1}^{1}, \alpha_{4,2}^{1}, \alpha_{4,3}^{1}, \alpha_{4,1}^{2}, \alpha_{4,2}^{2}, \alpha_{4,3}^{2}, \alpha_{4,1}^{3}, \alpha_{4,2}^{3}$ , $\alpha_{4,3}^{3}$. The initial conditions are:

$$
\begin{aligned}
& \alpha_{1, i}=\alpha_{2, i}=0 \quad(1 \leq i \leq 3), \\
& \alpha_{3, i}^{j}(0)=0, \quad \alpha_{4, i}^{j}(0)=1 \quad(1 \leq i, j \leq 3) .
\end{aligned}
$$

\section{Application of HAM: The -Curve and the Valid Region of Convergence of the Solutions}

In this section, we discuss the convergence of the HAM solutions. The convergence depends on the so-called convergence - control parameter $\hbar$, and so, we plot the $\hbar$-curve for $\theta_{g}(0), \eta(0), \theta_{d}(0)$, and $r_{d}(0)$. The interval of convergence is determined by the flat portion of the $\hbar$-curve. In order to plot the $\hbar$-curve we applied the HAM as given in [24] to our new model (21)-(24).

\section{An introduction to Homotopy Analysis Method (HAM)}

Consider the following differential equation:

$$
N(u(\vec{r}, t))=0 .
$$

where $N$ is a nonlinear operator, $\vec{r}$ is a vector of spatial variables, $t$ denotes time and $u$ is an unknown function.

Zero order deformation of HAM: By means of generalizing the traditional concept of homotopy, Liao [24] constructs the so-called zero-order deformation equation:

$$
(1-p) \ell\left[\Phi(\vec{r}, t ; p)-u_{0}(\vec{r}, t)\right]=\hbar H(\vec{r}, t) N(\Phi(\vec{r}, t ; p)),
$$

where $\hbar$ is a non-zero auxiliary parameter, $H$ is an auxiliary function, $\ell$ is an auxiliary linear operator, $u_{0}(\cdot)$ is an initial guess of $u(\cdot) ; \Phi$ is a unknown function. The degree of freedom is to choose the initial guess, the auxiliary linear operator, the auxiliary parameter, and the auxiliary function $H$. Expanding $\Phi$ in Taylor series with respect to the embedding parameter $p$, one has

$$
\Phi(\vec{r}, t ; p)=u_{0}(\vec{r}, t)+\sum_{n=1}^{\infty} u_{n}(\vec{r}, t) p^{n},
$$

where

$$
u_{n}(\vec{r}, t)=\left.\frac{1}{n !} \frac{\partial^{n} \Phi(\vec{r}, t ; p)}{\partial p^{n}}\right|_{p=0} .
$$

If the auxiliary linear operator, the initial guess, the auxiliary parameter, and the auxiliary function are so properly chosen that the above series converges at $p=1$, one has

$$
\Phi(\vec{r}, t ; p)=u_{0}(\vec{r}, t)+\sum_{n=1}^{\infty} u_{n}(\vec{r}, t)
$$

which must be one of the solutions of the original nonlinear equation, as proved in [24]. If the same initial guess and the same auxiliary linear operator are chosen, the approximations given by the homotopy perturbation method are exactly a special case of those given by the homotopy analysis method when $\bar{h}=-1$ and $H=1$. The series (54) itself is in principle a kind of Taylor series (at $\mathrm{p}=1)$. Hence, mathematically, homotopy perturbation method itself is also a kind of generalized Taylor technique.

mth-order deformation: Define the vector:

$$
\vec{u}_{n}(\vec{r}, t)=\left\{u_{0}(\vec{r}, t), u_{1}(\vec{r}, t), \ldots, u_{n}(\vec{r}, t)\right\} .
$$

Differentiating Equation (51) $m$-times with respect to the embedding parameter $p$ and then setting $p=0$ and finally dividing the terms by $m$ ! , we obtain the $m$ th-order deformation equation in the form of:

$$
\ell\left[u_{m}(\vec{r}, t)-\chi_{m} u_{m-1}(\vec{r}, t)\right]=\hbar H(\vec{r}, t) R_{m}\left(u_{m-1}(\vec{r}, t)\right),
$$

where,

$$
R_{m}\left(u_{m-1}(\vec{r}, t)\right)=\left.\frac{1}{(m-1) !} \frac{\partial^{m-1} N(\Phi(\vec{r}, t ; p))}{\partial p^{m-1}}\right|_{p=0},
$$

and $\chi_{m}$ is the unit step function. Applying the inverse operator $\ell^{-1}(\cdot)$ on both side of Equation (56), we get

$$
u_{m}(\vec{r}, t)=\chi_{m} u_{m-1}(\vec{r}, t)+\hbar \ell^{-1}\left[H(\vec{r}, t) R_{m}\left(u_{m-1}(\vec{r}, t)\right)\right] .
$$

In this way, it is easy to obtain $u_{m}$ for $m \geq 1$, at $m$ th-order and finally get the solution as:

$$
u(\vec{r}, t)=\sum_{n=0}^{m} u_{n}(\vec{r}, t) .
$$

In our model we choose the initial guess to be $\theta_{g}(0)=0, \eta(0)=1$ , $\theta_{d}(0)=0$, and $r_{d}(0)=1$ which satisfied the initial conditions. The linear operator will be:

$$
\ell=\frac{d}{d \tau}(\cdot)
$$

with the property $\ell\left(c_{1} \tau+c_{2}\right)=0$, where $c_{1}$ and $c_{2}$ are constants of integration. According to the system (21)-(24) and the terms as in Equation (32) the nonlinear operators will be defined as follows:

$$
N_{\theta_{g}}\left(\alpha_{1}(\tau, p)\right)=\frac{d \alpha_{1}}{d \tau}-\gamma^{-1} \alpha_{2} e^{\alpha_{1}}
$$


Citation: Nave O, Lehavi Y, Gol'dshtein V, Ajadi S (2012) Application of the Homotopy Perturbation Method (HPM) and the Homotopy Analysis Method (HAM) to the Problem of the Thermal Explosion in a Radiation Gas with Polydisperse Fuel Spray. J Appl Computat Math 1:115. doi:10.4172/2168-9679.1000115

Page 6 of 9

$$
\begin{gathered}
+\gamma^{-1} \sum_{i=1}^{k} \varepsilon_{1 i} \alpha_{4}^{i}\left(\alpha_{1}-\alpha_{3}^{i}\right)\left(1+\varepsilon_{4 i}(v-1) \alpha_{4}^{i}\right) \\
N_{\eta}\left(\alpha_{2}(\tau, p)\right)=\frac{d \alpha_{2}}{d \tau}+\alpha_{2} e^{\alpha_{1}} \\
-\psi \sum_{i=1}^{k} \varepsilon_{1 i} \alpha_{4}^{i}\left(\alpha_{1}-\alpha_{3}^{i}\right)\left(1+\varepsilon_{4 i}(v-1) \alpha_{4}^{i}\right) \\
N_{\theta_{d_{i}}}\left(\alpha_{3}^{i}(\tau, p)\right)=\frac{d \alpha_{3}^{i}}{d \tau} \\
-\varepsilon_{3 i} \alpha_{4}^{i}\left(\alpha_{1}-\alpha_{3}^{i}\right)\left(1+\varepsilon_{4 i}(v-1) \alpha_{4}^{i}\right) \\
1 \leq i \leq k \\
N_{r_{d_{i}}}\left(\alpha_{4}^{i}(\tau, p)\right)=\frac{d\left(\alpha_{4}^{i}\right)^{3}}{d \tau} \\
+\varepsilon_{1 i} \varepsilon_{2 i} \alpha_{4}^{i}\left(\alpha_{1}-\alpha_{3}^{i}\right)\left(1+\varepsilon_{4 i}(v-1) \alpha_{4}^{i}\right) \\
1 \leq i \leq k
\end{gathered}
$$

By substituting the series (32) into Equations (61)-(65) correspondingly we get the terms for $R_{m}$ accordingly to Equation (57) as follows:

$$
\begin{aligned}
& \gamma R_{m_{\theta_{g}}}=\gamma \frac{d \alpha_{1, m-1}}{d \tau}-\alpha_{2, m}-\sum_{n=0}^{m-1} \alpha_{2, n} \alpha_{1, m-1-n} \\
& -0.5 \sum_{n=0}^{m-1} \alpha_{2, m-1-n}^{i} \sum_{j=0}^{n} \alpha_{1, j} \alpha_{1, n-j} \\
& +\sum_{i=1}^{k}\left(\sum_{n=0}^{m-1} \varepsilon_{1 i}\left(\alpha_{4, n}^{i} \alpha_{1, m-1-n}-\alpha_{4, n}^{i} \alpha_{3, m-1-n}^{i}\right)\right) \\
& +\sum_{i=1}^{k}\left(\varepsilon_{1 i} \varepsilon_{4 i}\left(\sum_{n=0}^{m-1} \alpha_{4, m-1-n}^{i} \sum_{j=0}^{n} \alpha_{1, j} \alpha_{4, n-j}^{i}\right)\right) \\
& -\sum_{i=1}^{k}\left(\varepsilon_{1 i} \varepsilon_{4 i}\left(\sum_{n=0}^{m-1} \alpha_{4, m-1-n}^{i} \sum_{j=0}^{n} \alpha_{3, j}^{i} \alpha_{4, n-j}^{i}\right)\right), \\
& R_{m \eta}=\frac{d \alpha_{2, m-1}}{d \tau}+\alpha_{2, m-1}+\sum_{n=0}^{m-1} \alpha_{2, n} \alpha_{1, m-1-n} \\
& +0.5 \sum_{n=0}^{m-1} \alpha_{2, m-1-n}^{i} \sum_{j=0}^{n} \alpha_{1, j} \alpha_{1, n-j} \\
& -\psi \sum_{i=1}^{k}\left(\sum_{n=0}^{m-1} \varepsilon_{1 i}\left(\alpha_{4, n}^{i} \alpha_{1, m-1-n}-\alpha_{4, n}^{i} \alpha_{3, m-1-n}^{i}\right)\right) \\
& -\psi \sum_{i=1}^{k}\left(\varepsilon_{1 i} \varepsilon_{4 i}\left(\sum_{n=0}^{m-1} \alpha_{4, m-1-n}^{i} \sum_{j=0}^{n} \alpha_{1, j} \alpha_{4, n-j}^{i}\right)\right) \\
& +\psi \sum_{i=1}^{k}\left(\varepsilon_{1 i} \varepsilon_{4 i}\left(\sum_{n=0}^{m-1} \alpha_{4, m-1-n}^{i} \sum_{j=0}^{n} \alpha_{3, j}^{i} \alpha_{4, n-j}^{i}\right)\right), \\
& R_{m_{\theta_{d_{i}}}}=\frac{d \alpha_{3, m-1}^{i}}{d \tau}
\end{aligned}
$$

Now, the solution of the $m$ th-order deformation can be expressed according to Equation (58) which can be solved by symbolic software such as Mathematica 8.0, Maple, Matlab and so on. We obtain a family of solutions that depends on the auxiliary parameter $\hbar$. So, regarding $\hbar$ as independent variable, it is easy to plot the $\hbar$-curves. For example, we can plot the curves:

$$
\Gamma_{1}=u(\vec{r}, t)_{\mid \vec{r}=0, t=0}, \text { or } \quad \Gamma_{2}=u^{\prime}(\vec{r}, t)_{\mid \vec{r}=0, t=0}, \text { or } \quad \Gamma_{3}=u^{\prime \prime}(\vec{r}, t)_{\mid \vec{r}=0, t=0} .
$$

The curves $\Gamma_{i}(i=1,2,3)$ are a function of $\hbar$ and thus can be plotted by a curve $\Gamma \approx \hbar$. According to [20] there exists a horizontal line segment (flat portion of the $\hbar$-curve) in the figure of $\Gamma \approx \hbar$ and called the valid region of $\hbar$ which corresponds to a region of convergent of the solutions. Thus, if we choose any value in the valid region of $\hbar$ we are sure that the corresponding solutions series are convergent. For given initial approximation $u_{0}(\vec{r}, t)$, the auxiliary linear operator $\ell$, and the auxiliary function $H(\vec{r}, t)$, the valid region of $\hbar$ for different special quantities are often nearly the same for a given problem. Hence, the so-called $\hbar$-curve provides us a convenient way to show the influence of $\hbar$ on the convergence region of the solutions series.

\section{Discussion and Conclusions}

We compared the system dynamics of the models (21)-(24) and (37)-(48) with and without the impact of the thermal radiation. The results are based on the following diesel engines parameter values:

Diesel-engines

$$
\begin{aligned}
& c_{p g}=1120 \quad\left(\begin{array}{ll}
J & k^{-1} K^{-1}
\end{array}\right) ; \quad \rho_{g o}=23.8 \quad\left(\begin{array}{ll}
k g & m^{-3}
\end{array}\right) ; \\
& k_{11}=0.08 ; \quad k_{01}=0.28 ; \quad \sigma=5.67 \times 10^{-8}\left(\mathrm{Wm}^{-2 k^{-4}}\right) \text {; }
\end{aligned}
$$


Citation: Nave O, Lehavi Y, Gol'dshtein V, Ajadi S (2012) Application of the Homotopy Perturbation Method (HPM) and the Homotopy Analysis Method (HAM) to the Problem of the Thermal Explosion in a Radiation Gas with Polydisperse Fuel Spray. J Appl Computat Math 1:115. doi:10.4172/2168-9679.1000115

$$
\begin{aligned}
& \mu_{f}=170 \quad\left(\mathrm{~kg} \quad \mathrm{kmol}^{-1}\right) \text {; } \\
& Q_{f}=4.3 \cdot 10^{7} \quad\left(J \quad \mathrm{~kg}^{-1}\right) ; \quad E=1.26 \cdot 10^{8} \quad\left(\mathrm{Jkg}^{-1}\right) ; \\
& \lambda_{g 0}=0.061 \quad\left(\mathrm{Wm}^{-1} \mathrm{~K}^{-1}\right) ; \quad \alpha_{g}=1 \quad \text { (dimensionless); } \\
& R_{d i 0}=5 \times 10^{-4} \quad(\mathrm{~m}) ; 10 \times 10^{-4} \quad(\mathrm{~m}) ; 15 \times 10^{-4} \quad(\mathrm{~m}) ; \\
& n_{d i}=8 \times 10^{11} \quad(\mathrm{~m}) ; 12 \times 10^{12} \quad(\mathrm{~m}) ; 16 \times 10^{14} \quad(\mathrm{~m}) ; \\
& A=3 \times 10^{6} \quad\left(s^{-1}\right) ; \quad T_{d 0}=300 \quad(K) ; \\
& L=3.6 \cdot 10^{5} \quad\left(\begin{array}{ll}
J & k^{-1}
\end{array}\right) ; \quad T_{g 0}=900 \quad(K) ; \\
& \gamma=2.184 \times 10^{-4}, \quad \beta=9.84 \times 10^{-2}, \quad \psi=1.19 \times 10^{2} \text {; } \\
& \varepsilon_{11}=3.7 \times 10^{-3}, \quad \varepsilon_{12}=4.73 \times 10^{-3}, \quad \varepsilon_{13}=5.73 \times 10^{-3} ; \\
& \varepsilon_{21}=6.9 \times 10^{1}, \quad \varepsilon_{22}=7.1 \times 10^{1}, \quad \varepsilon_{23}=7.5 \times 10^{1} \text {; } \\
& \varepsilon_{31}=3.71 \times 10^{-1}, \quad \varepsilon_{32}=4.1 \times 10^{-1}, \quad \varepsilon_{33}=4.8 \times 10^{-1} \text {; } \\
& \varepsilon_{41}=2.5 \times 10^{-2}, \quad \varepsilon_{42}=3 \times 10^{-2}, \quad \varepsilon_{43}=3.5 \times 10^{-2} \text {. }
\end{aligned}
$$

We studied the problem of the the effect of fuel spray polydispersity on the ignition process in a fuel cloud by applying numerical simulation, the homotopy perturbation method and the homotopy analysis method for 30th order deformation. We compared between the homotopy perturbation method and by solving the full system of the model i.e., the system of Equations: (21)-(24) for the solution profiles of the of the gas temperature, droplet temperature, radius and concentration numerically. Although we take into account only three different size of droplets, our results show that the homotopy perturbation method provides an excellent approximation of the solutions of the system with high accuracy (Figures 1-4).

The gas temperature trajectory, figure 1 , for all models with and

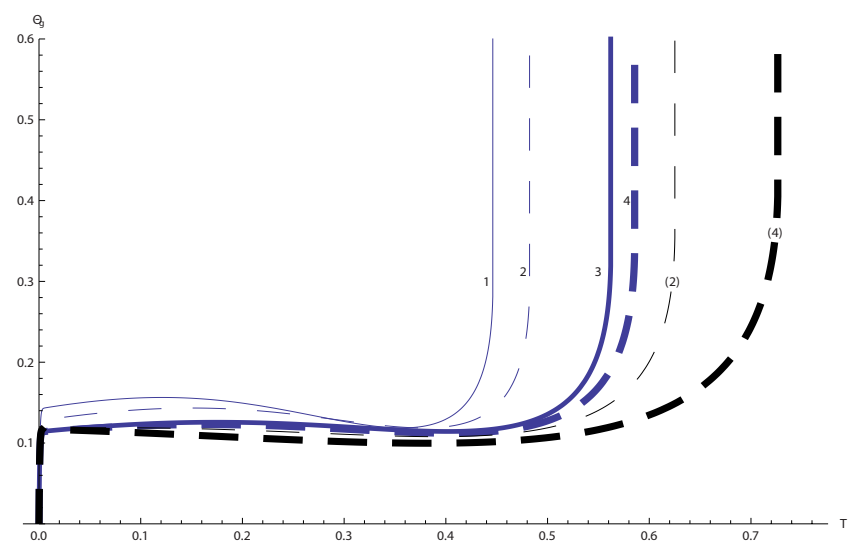

Figure 1: Solution profiles of the gas temperature $\theta_{g}-\tau ; 1$ : Full model solved numerically with the impact of the thermal radiation, 2 : HPM model with the impact of the thermal radiation for the $\hbar=-1,(2)$ : HAM model with the impact of the thermal radiation for the $\hbar=0.04,3$ : Full model solved numerically without the impact of the thermal radiation, 4: HPM model without the impact of the thermal radiation, (4): HAM model without the impact of the thermal radiation for $\hbar=0.04$.

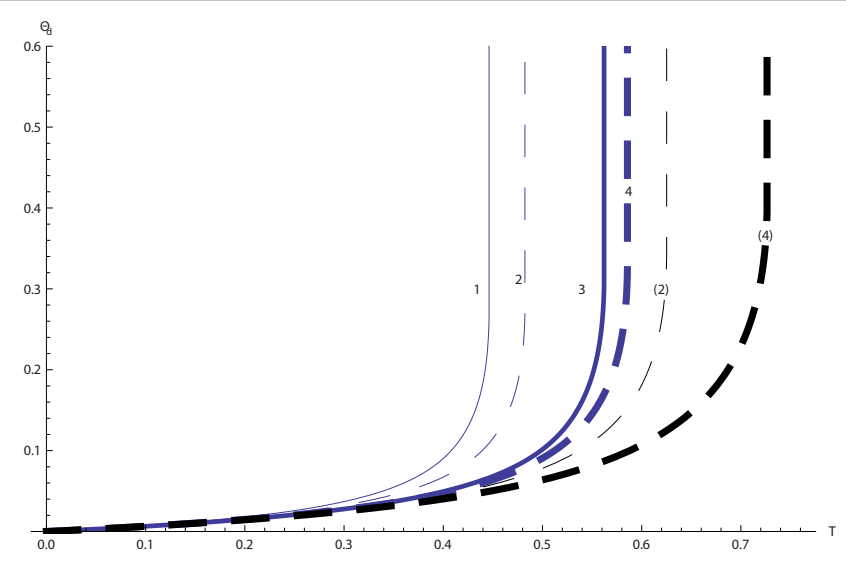

Figure 2: Solution profiles of the droplet temperature $\theta_{d}-\tau ; 1$ : Full model solved numerically with the impact of the thermal radiation, 2: HPM model with the impact of the thermal radiation for the $\hbar=-1,(2)$ : HAM model with the impact of the thermal radiation for the $\hbar=0.04,3$ : Full model solved numerically without the impact of the thermal radiation, 4: HPM model without the impact of the thermal radiation, (4): HAM model without the impact of the thermal radiation for $\hbar=0.04$.

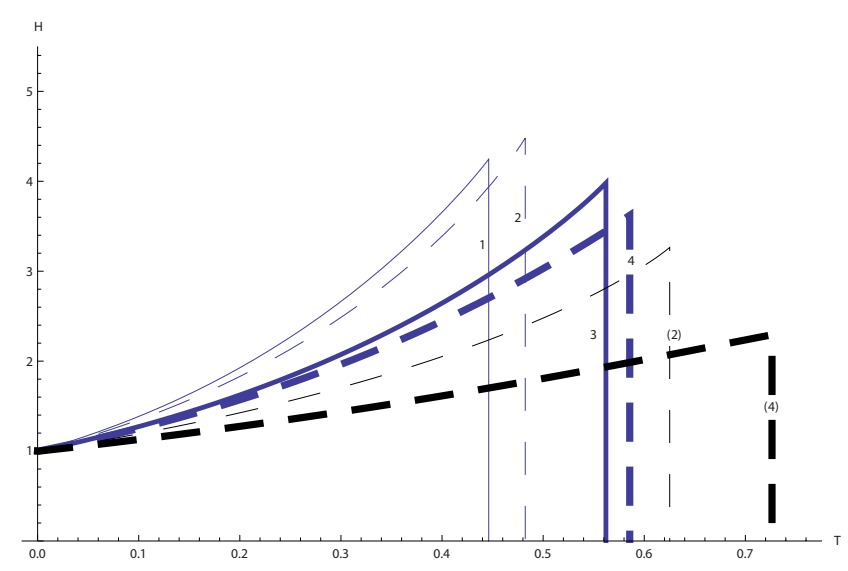

Figure 3: Solution profiles of the radius $r-\tau ; 1$ : Full model solved numerically with the impact of the thermal radiation, 2 : HPM model with the impact of the thermal radiation for the $\hbar=-1,(2)$ : HAM model with the impact of the thermal radiation for the $\hbar=0.04,3$ : Full model solved numerically without the impact of the thermal radiation, 4: HPM model without the impact of the thermal radiation, (4): HAM model without the impact of the thermal radiation for $\hbar=0.04$.

without the impact of the thermal radiation, starts with a fast increase in the temperature until $\theta_{g} \approx 0.15$ then the temperature decreases from $\theta_{g} \approx 0.15$, which means cooling before ignition, until $\theta_{g} \approx 0.1$. This continuous process of cooling before ignition is summarized in table 1 and corresponding in figures 1-4. This dimensionless time, $\tau$, refers to the ignition time and it is compatible for all the solution profiles for the gas and droplets temperature, radius and concentration. According to these results, the $F-M$ has the smallest ignition time with and without the impact of the thermal radiation when comparing to HPM and HAM. The HPM results are closer to the $F-M$ than the HAM results with and without the impact of the thermal radiation.

The presence of the small parameter $\gamma$ in the gas temperature equations, such that Equations (37)-(39) form a singularly perturbed system, enables one to exploit the geometrical version of the method of the integral manifold and hence to separate the model into fast and 
Citation: Nave O, Lehavi Y, Gol'dshtein V, Ajadi S (2012) Application of the Homotopy Perturbation Method (HPM) and the Homotopy Analysis Method (HAM) to the Problem of the Thermal Explosion in a Radiation Gas with Polydisperse Fuel Spray. J Appl Computat Math 1:115. doi:10.4172/2168-9679.1000115

Page 8 of 9

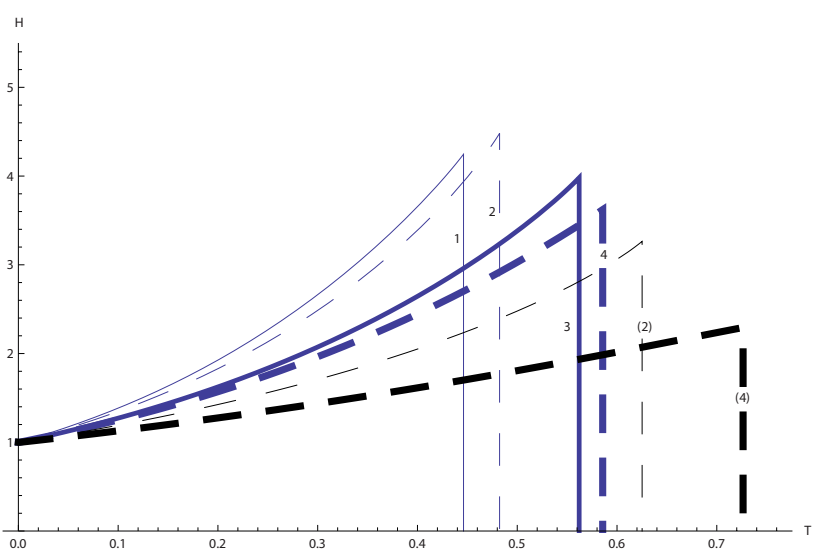

Figure 4: Solution profiles of the concentration $\eta-\tau$; 1 : Full model solved numerically with the impact of the thermal radiation, 2: HPM model with the impact of the thermal radiation for the $\hbar=-1,(2)$ : HAM model with the impact of the thermal radiation for the $\hbar=0.04,3$ : Full model solved numerically without the impact of the thermal radiation, 4: HPM model without the impact of the thermal radiation, (4): HAM model without the impact of the thermal radiation for $\hbar=0.04$

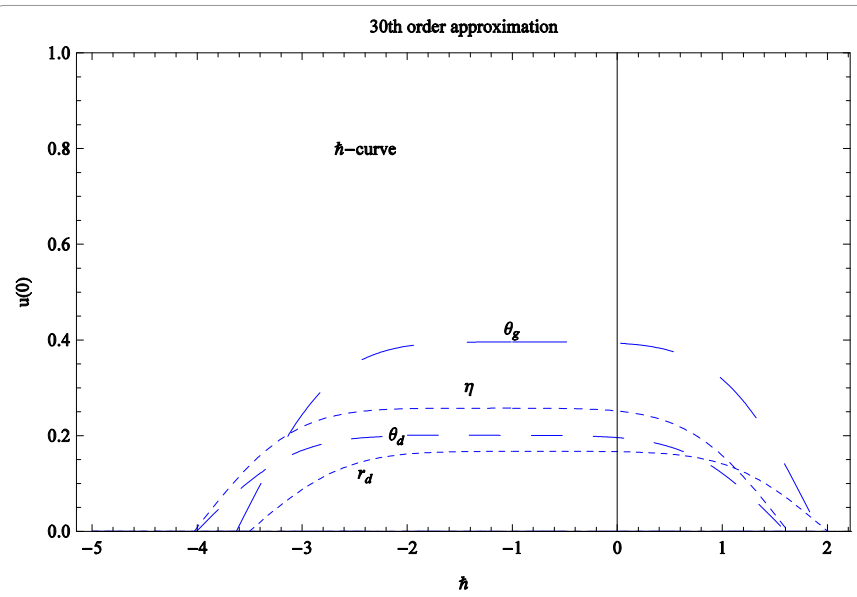

Figure 5: Plot curve for the 30th order approximation.

\begin{tabular}{|l|l|l|}
\hline Model & radiation & no-radiation \\
\hline 1: Full-model & $=0.446306$ & $=0.562209$ \\
\hline 2: HPM & $=0.482483$ & $=0.585683$ \\
\hline 3: HAM & $=0.625222$ & $=0.726507$ \\
\hline
\end{tabular}

Table 1: The process of cooling before the ignition time for the different models. $F-M$ refer to the full-model, HPM with $\hbar=-1$ and HAM with $\hbar=0.04$

\begin{tabular}{|c|c|}
\hline Parameter & \\
\hline 1:_rad ${ }^{\wedge}=0.04=\left|{ }_{1} \mathrm{HAM}^{\wedge}=0.04-\_F-M\right|$ & 0.17891 \\
\hline 2:_rad ra $^{\wedge}=-1=\left|\_H P M^{\wedge}=-1-\_F-M\right|$ & 0.03617 \\
\hline 3:_no-rad ${ }^{\wedge}=0.04=\left|\_H_{A M}^{\wedge}=0.04-\_F-M\right|$ & 0.16429 \\
\hline 4:_no-rad ${ }^{\wedge}=-1=\mid{ }_{1} \mathrm{HPM}^{\wedge}=-1-$ F-M $\mid$ & 0.02347 \\
\hline
\end{tabular}

Table 2: The impact of the convergence-control parameter $\hbar$ on the solutions profiles with and without the impact of the thermal radiation comparing to the numerical simulations.

slow subsystems. According to this method, the above results are also sustained with MIM [25].

In order to clarify the influence of the thermal radiation on the dimensionless time before the final explosion of the system we introduce the term impact of the thermal radiation as given in [26] which is measured in percent and defined as:

$$
\begin{aligned}
& \Delta_{1}(\%)=\frac{\left|\tau_{F-M}^{\mathrm{rad}}-\tau_{F-M}^{\text {no-rad }}\right|}{\tau_{F-M}^{\mathrm{rad}}} \cdot 100, \\
& \Delta_{2}(\%)=\frac{\left|\tau_{H P M}^{\mathrm{rad}}-\tau_{H P M}^{\text {no-rad }}\right|}{\tau_{H P M}^{\mathrm{rad}}} \cdot 100, \\
& \Delta_{3}(\%)=\frac{\left|\tau_{H A M}^{\mathrm{rad}}-\tau_{H A M}^{\text {no-rad }}\right|}{\tau_{\text {HAM }}^{\text {rad }}} \cdot 100,
\end{aligned}
$$

where the subscript $F-M$ refers to the full model solved numerically, $\tau$ is the ignition time, and the superscript $\mathrm{rad}$ and no-rad refer to the model with and without the impact of the thermal radiation respectively. We also defined the parameters $\left|\Delta_{1}-\Delta_{2}\right|$ , $\left|\Delta_{1}-\Delta_{3}\right|$, and $\left|\Delta_{2}-\Delta_{3}\right|$ which show the difference between the different models in percent. The results are as follows: $\Delta_{1}=25.969 \%$, $\Delta_{2}=21.389 \%, \Delta_{3}=16.199 \%$ and $\left|\Delta_{1}-\Delta_{2}\right|=4.58 \%,\left|\Delta_{1}-\Delta_{3}\right|=9.77 \%$ , and $\left|\Delta_{2}-\Delta_{3}\right|=5.19 \%$. As we can see from these results, the HPM model is closer to the model solved with numerical simulations than the HAM model (the comparison between the $F-M$ and the HPM model based on the value of $\hbar$ as equal to -1 , and the comparison between the $F-M$ and the HAM model based on the value of $\hbar$ as equal to 0.04 ).

As we mentioned in the previous section, the convergence depends on the convergence-control-parameter $\hbar$, and so we plot the $\hbar$-curve for $\theta_{g}(0), \theta_{d}(0), \eta(0)$ and $r_{d}(0)$ as shown in figure 5 for $m=30$ in Equation (59) i.e. 30 th order approximation. According to figure 5 , the interval of convergence that agrees for all of the corresponding solutions is $\hbar \in[-1.87,0.05]$. In order to emphasize the impact of the convergence-control parameter $\hbar$ on the solutions profiles we defined the terms:

$$
\begin{aligned}
& \sum_{\text {rad }}^{\hbar=0.04}=\left|\tau_{H A M}^{\hbar=0.04}-\tau_{F-M}\right|, \\
& \sum_{\text {rad }}^{\hbar=-1}=\left|\tau_{H P M}^{\hbar=-1}-\tau_{F-M}\right|, \\
& \sum_{n o-\text { rad }}^{\hbar=0.04}=\left|\tau_{H A M}^{\hbar=0.04}-\tau_{F-M}\right|, \\
& \sum_{\text {no-rad }}^{\hbar=-1}=\left|\tau_{H P M}^{\hbar=-1}-\tau_{F-M}\right|,
\end{aligned}
$$

which point out the difference (in dimensionless-time) between the profiles solutions of the HPM and HAM methods for different $\hbar$ with the impact of the thermal radiation and without the impact of the thermal radiation respectively from the numerical results, and $\tau$ refer to the ignition time. The results are summarized in table 2. According to these results, the HPM and the HAM methods are closed to the numerical results for both with and without the impact of the thermal radiation.

We have shown the the solutions obtained by HPM and HAM are convergent and that they extremely well with numerical simulations. It has also been shown that the homotopy perturbation method, which is a special case of the homotopy analysis method when $\hbar=-1$, yields 
Citation: Nave O, Lehavi Y, Gol'dshtein V, Ajadi S (2012) Application of the Homotopy Perturbation Method (HPM) and the Homotopy Analysis Method (HAM) to the Problem of the Thermal Explosion in a Radiation Gas with Polydisperse Fuel Spray. J Appl Computat Math 1:115. doi:10.4172/2168-9679.1000115

Page 9 of 9

convergent solutions for all of the cases considered. These results demonstrate that HPM and HAM are very effective analytical methods for solving nonlinear problems in science and engineering.

Our next step in this direction is to apply the HPM to the continuous model as in our previous work $[27,28]$.

\section{References}

1. Aggrawal SK (1998) A review of spray ignition phenomena: present status and future research. Prog Energy Combust Sci 24: 565-600

2. Babushok VI, Gol'dshtein V (1988) Structure of the thermal explosion limit. Combust and Flame 72: 221-226.

3. Gol'dshtein VM, Sobolev VA (1992) Singularity theory and some problems of functional analysis.American Math Soci 153: 73-92.

4. Sazhin SS, Feng G, Heikal MR (1992) A model for fuel spray penetration. Fue 80: $2171-2180$

5. Liao SJ (1992) The proposed homotopy analysis technique for the solution of nonlinear problems. Ph.D Thesis Shanghai Jiao Tong University.

6. He JH (1998) Approximate analytical solution for seepage flow with fractiona derivatives in porous media. Comput Meth Appl Mech Eng 16: 57-68.

7. Liao SJ (2005) Comparison between the homotopy analysis method and homotopy perturbation method. Appl Math Comp169: 1186-1194.

8. Liao SJ (2007) A short communication on Dr. He's modified Lindstedt-Poincare method NONLINEAR DYNAMICS 49: 317-318.

9. Pakdemirli M, Karahan MMF, Boyac H (2009) A new perturbation algorithm with better convergence properities: multiple scales Lindstedt-Poincare method Math Comp App 14: 31-44.

10. Hu H, Xiong ZG (2004) Comparison of two Lindstedt-Poincare type perturbation methods. J Sound and Vibration 278: 437-444.

11. He JH (2004) The homotopy perturbation method for non-linear oscillators with discontinuities. Appl Math Comput 151: 287-292.

12. He JH (2005) Application of homotopy perturbation method to nonlinear wave equations. Chaos Solitons Fractals 26: 695-700.

13. He JH (2006) Homotopy perturbation method for solving boundary problems Phys Lett A 350: 87-88.
14. He JH (2005) Limit cycle and bifurcation of nonlinear problems. Chaos Solitons Fractals 26: 827-833

15. He JH (2004) Asymptotology by homotopy perturbation method. Appl Math Comput 156: 591-596.

16. He JH (2005) Limit cycle and bifurcation of nonlinear problems. Chaos Solitons Fractals 26: 827-833.

17. He JH (2005) Homotopy-perturbation method for bifurcation of nonlinear problems. Internat J Nonlinear Sci Numer Simul 6: 207-208.

18. Liao SJ (2004) On the homotopy analysis method for nonlinear problems. App Math Comp 147: 499-513.

19. Liao SJ (2009) Notes on the homotopy analysis method: Some definition and theorems. Comm in Nonlinear Sci Numerical Simu 14: 983-997.

20. Goldfarb I, Gol'dshtein V, Zinoviev A (2002) Delayed thermal explosion in porous media: Method of invariant manifolds. IMA J App Math 67: 263-280.

21. Frank DA-Kamenetskii (1969) Diffusion and heat exchange in chemical kinetics. Plenum Press New York: USA.

22. Ajadi SO, Zuilino M (2011) Approximate analytical solutions of reactiondiffusion equations with exponential source term: Homotopy perturbation method (HPM). App Math Let 24: 1634-1639.

23. Turgut $O$, Ahmet $Y$ (2008) Comparison between Adomian's method and He's homotopy perturbation method. Comp Math App 56: 1216-1224.

24. Liao SJ (2003) Beyond Perturbation: Introduction to the Homotopy Analysis Method. Chapman and Hall/CRC Press: Boca Raton.

25. Goldfarb I, Gol'dshtein V, Greenberg BJ, Kuzmenko G (2002) Analysis of Radiative Heat-Loss Effects in Thermal Explosion of a Gas using the Integra Manifold Methods. J Engng Math 44: 229-243.

26. Goldfarb, Gol'dshtein VM, Katz D, Sazhin S (2007) Radiation effect on therma explosion in a gas containing evaporation fuel droplets. Int Journal of Thermal Sci 46: 358-370.

27. Nave O, Gol'dshtein VM , Vitcheslav B (2010) A probabilistic model of therma explosion in polydisperse fuel spray. Applied Math Comp 217: 2698-2709.

28. Nave O, Gol'dshtein VM (2011) The flammable spray effect on therma explosion of combustible gas-fuel mixture. Combus Sci Tech 183: 519-539. 